\title{
Performance Assessment for the Feedforward-Cascade Drum Water Level Control System
}

\author{
Zhi Zhang*1,2 \\ 1. Postdoctoral Programme of China Industrial Security Research Center, Beijing \\ Jiaotong University, Beijing, 100044, China \\ 2. School of Electrical Engineering, Beijing Jiaotong University, Beijing, 100044, \\ China \\ zhizhang@bjtu.edu.cn
}

\begin{abstract}
The boiler drum water level control system is one of the most important components of a power plant. The operating water level control system is increasingly recognized as a capital asset that should be routinely maintained and monitored. The performance assessment technology can achieve measures of the level of difficulty in controlling the water level from routine operating data, however, which is still an open problem; thus, in this paper the performance assessment technology will be brought into the feedforwardcascade drum water level control system. The performance benchmark and the associated benchmark controller are also proposed. A numerical example for the drum water level control system shows the effectiveness of the proposed method.
\end{abstract}

Keywords: Performance assessment; Feedforward-cascade control; Drum water level control system

\section{Introduction}

The water level in a boiler drum is an important index for the safe and steady operation of an industrial steam boiler. It should be controlled in safe limits in order to maintain plant availability and economic feasibility of a power plant. Hence, a suitable controller of the boiler drum water level control systems is the crucial for the power plants. Although a variety of control design techniques (e.g., PID, model predictive, fuzzy logic, etc.) were considered in the design of the level controller $[1,2]$, fewer techniques exist for objective measures of water level control loop performance or, conversely, measures of the level of difficulty in controlling the water level from routine operating data.

Unlike the controller design problem which focuses on developing the control strategy, the controller performance assessment problem is concerned about whether the designed controller performance is in accordance with the required performance in the design stage. Many factors can contribute to their performance deterioration, including sensor or actuator failure, equipment fouling, feedstock variability, product changes, and seasonal influences [3]. Hence, it is necessary to offer control engineers a well-suited tool for determining whether specified performance targets and response characteristics are being met by the controlled process variables [4]. In the past two decades, research works on control loops performance assessment have attracted growing interest.

By virtue of valuable contributions from Harris, time series analysis techniques could be used to find a suitable expression for the feedback controller invariant from routine operating data and the subsequent use of this as a benchmark to assess control loop performance [5]. The associated minimum variance (MV) controller, as a benchmark controller, can also be obtained. A well-established performance benchmark can be used to test the deviation from the current control performance. Subsequently, the MV 
performance benchmark was extended to the multivariable control loop performance assessment [6]. Some excellent works on the control loop performance assessment can be found in the literature [4] and references therein.

Based on the above considerations, the work presented in this paper aims to develop the controller performance assessment method for the feedforward-cascade drum water level control system. The performance benchmark and the associated benchmark controller will be proposed. The remainder of this paper is organized as follows. Section 2 presents the plant description and the considered drum water level control system. In terms of the feedforward-cascade controller systems, the performance assessment method for the plant is developed in Section 3. Section 4 presents the numerical example that shows the effectiveness of the proposed method. Conclusions are stated in Section 5.

\section{Drum Water Level Control System Description}

The water level control system of boiler drum widely used in industry field is a cascade three impulse control system. In fact, it is a kind of feedforward-cascade control system. As shown in Figure 1, it consists of the master controller, the secondary controller, and the three impulses. The drum water level, the stream flow rate and the feed water rate are the three impulses, which are denoted by $H, D$ and $W$, respectively. The Controller 1 which is the main controller is for water level control. The Controller 2 which is the secondary controller is for feed water flow rate control. The steam flow signal is used as a feed forward signal to maintain the material balance when the load is changed. $\Delta p$ is the differential pressure transmitter. $\sqrt{ }$ is the square root extractor.

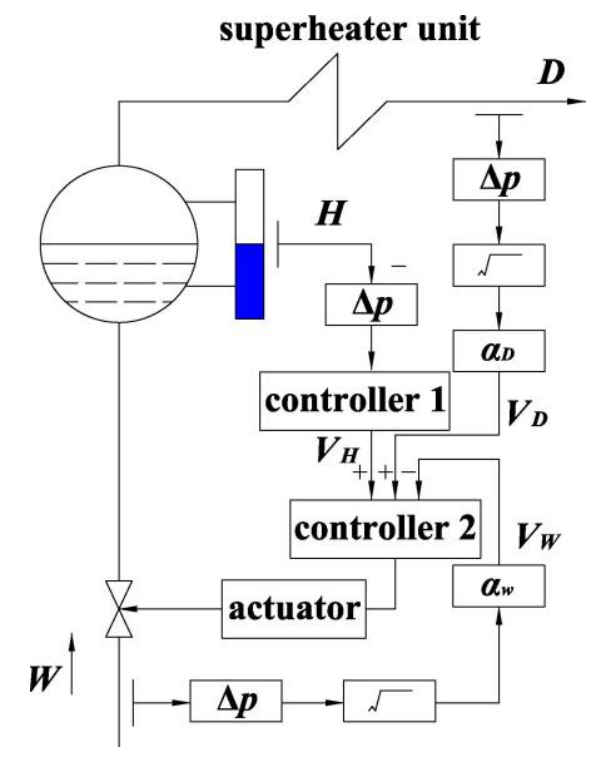

Figure 1. Drum Water Level Control System

After discretization, the control system principle diagram is shown in Figure 2. $G_{P}$ is the plant transfer function. $\gamma_{H}$ is the water level measurement transmitter. $\gamma_{W}$ is the feed water flow rate transmitter. $\gamma_{D}$ is the stream flow rate measurement transmitter. $\alpha_{W}$ is the feedback device of the feed water flow rate. $\alpha_{D}$ is the feedforward device of the stream flow rate. $K_{Z}$ is the actuator. $K_{u}$ is the control valve. The secondary control loop is consists of $\gamma_{W}, \alpha_{W}, G_{C 2}, K_{Z}$ and $K_{u}$. The main control loop is consists of the secondary loop and the main controller $G_{C 1}$. 


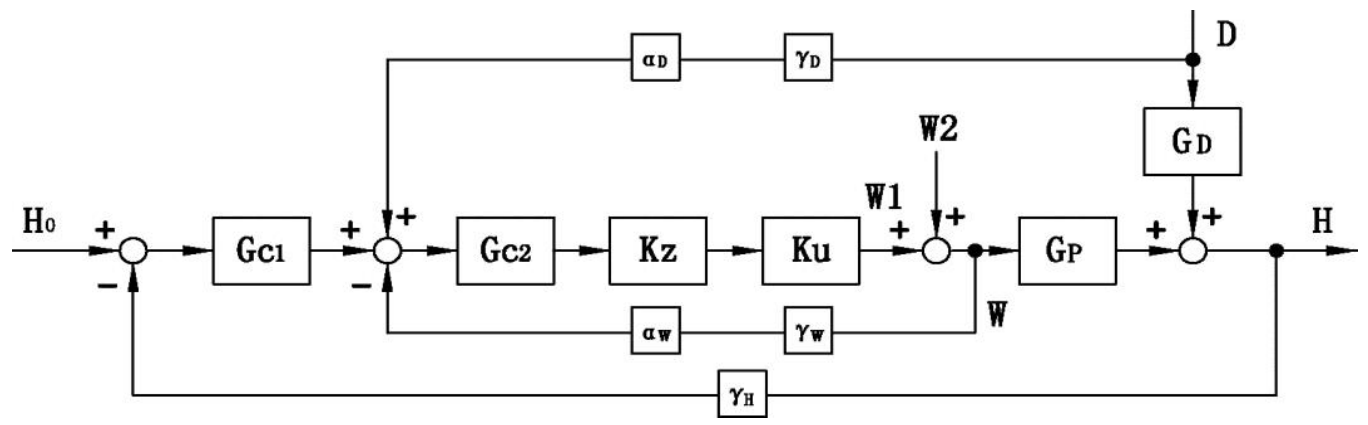

Figure 2. Discretized Drum Water Level Control System

\section{Performance Assessment for the Drum Water Level Control System}

\subsection{Performance Benchmark of the Drum Water Level Control System}

For the whole control loop, the measurable disturbance in the main loop can be equivalent to the additive disturbance at output of the main loop. The immeasurable disturbance in the main loop and secondary loop can be equivalent to the additive disturbance at output of the main loop and secondary loop, respectively. Thus, the Figure 2 can be rewritten as the Figure 3. Then, we have

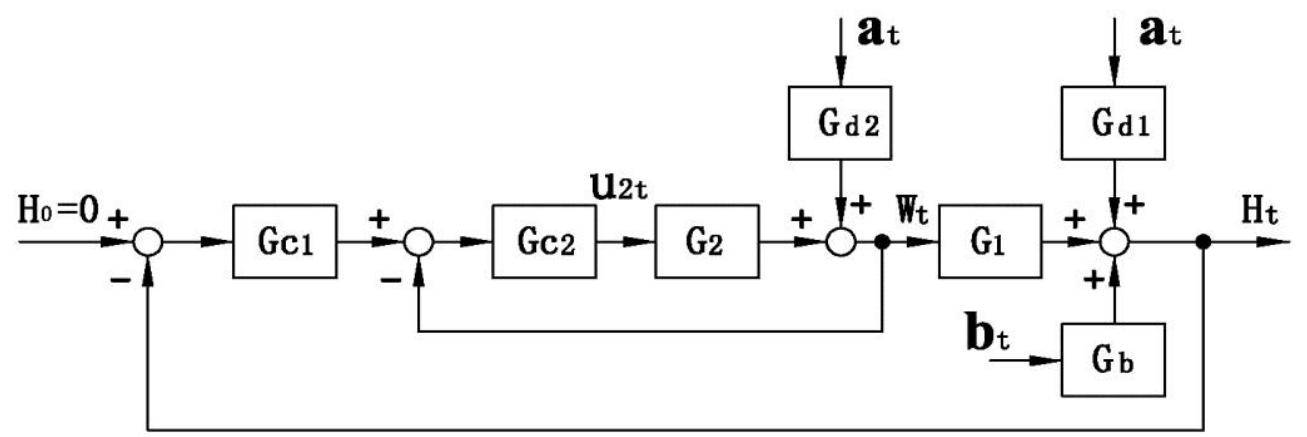

Figure 3. Equivalent Discretized Drum Water Level Control System

and

$$
\begin{aligned}
H_{t} & =G_{1} W_{t}+G_{d 1} a_{t}+G_{b} b_{t} \\
W_{t} & =G_{2} u_{2 t}+G_{d 2} a_{t} \\
u_{2 t} & =-G_{C 2}\left(W_{t}+H_{t} G_{C 1}\right),
\end{aligned}
$$

$$
H_{t}=\frac{\left(\left(1+G_{2} G_{C 2}\right) G_{d 1}+G_{1} G_{d 2}\right) a_{t}+\left(1+G_{2} G_{C 2}\right) G_{b} b_{t}}{1+G_{2} G_{C 2}+G_{1} G_{2} G_{C 1} G_{C 2}}
$$

where $H_{t}$ is the deviation of water level output from its set-point, and $W_{t}$ is the deviation of feed water flow rate output from its steady state value. $G_{1}$ denotes the transfer function from feed water flow rate to water level, where $G_{1}\left(q^{-1}\right)=\bar{G}_{1} q^{-d_{1}}, q^{-d_{1}}$ is a $d_{1}$-step time delay, $\overline{G_{1}}$ is the delay-free transfer function. $G_{2}$ denotes the transfer function from the sum of the output of the main controller and the secondary controller to feed water 
flow rate, $G_{2}\left(q^{-1}\right)=\overline{G_{2}} q^{-d_{2}}, q^{-d_{2}}$ is a $d_{2}$-step time delay, and $\overline{G_{2}}$ is the delay-free transfer function. Assume that $G_{1}$ and $G_{2}$ are the minimum phase. The disturbance transfer functions, $G_{d 1}$ and $G_{d 2}$ are assumed to be the rational function of $q^{-1}$, and they are driven by the Gaussian white noise sequences $a_{t}$ with zero mean and variance $\sigma_{a}^{2}$. $G_{b}$ denotes the transfer function from steam flow rate disturbance to water level. Assume that $\xi_{t}$ is the measurable disturbance, and it is driven by the Gaussian white noise sequences $b_{t}$ with zero mean and variance $\sigma_{b}^{2} . a_{t}$ and $b_{t}$ are independent of each other. Then, we can obtain $\xi_{t}=G_{m} b_{t}$, where $G_{m}$ is the transfer function from $b_{t}$ to $\xi_{t}$, which can be estimated by time series analysis. Thus, the equation (2) can be represented as

$$
H_{t}=G_{a} a_{t}+G_{\xi} \xi_{t}
$$

where

$$
G_{a}=\frac{\left(\left(1+G_{2} G_{C 2}\right) G_{d 1}+G_{1} G_{d 2}\right)}{1+G_{2} G_{C 2}+G_{1} G_{2} G_{C 1} G_{C 2}}, \quad G_{\xi}=\frac{\left(1+G_{2} G_{C 2}\right) G_{b} G_{m}^{-1}}{1+G_{2} G_{C 2}+G_{1} G_{2} G_{C 1} G_{C 2}} .
$$

There are Diophantine identities as follows:

$$
\left\{\begin{aligned}
G_{b} & =F_{b}+R_{b} q^{-d_{1}-d_{2}} \\
G_{d 1} & =F_{1}+R_{1} q^{-d_{1}-d_{2}} \\
G_{d 2} & =F_{2}+R_{2} q^{-d_{2}} \\
\overline{G_{1}} F_{2} & =S+T_{T} q^{-d_{2}}
\end{aligned}\right.
$$
polynomials in $q^{-1}$ of the order $d_{2}-1$, and $R_{b}, R_{1}, R_{2}$ and $T$ are proper transfer functions.

Substituting (4) to (2), we have

$$
\begin{aligned}
H_{t} & =F_{b} b_{t}+\left(F_{1}+S q^{-d_{1}}\right) a_{t}+q^{-d_{1}-d_{2}}\left[\frac{\left(1+G_{2} G_{C 2}\right) R_{b}-F_{b} \overline{G_{1}} \overline{G_{2}} G_{C 1} G_{C 2}}{1+G_{2} G_{C 2}+G_{1} G_{2} G_{C 1} G_{C 2}}\right] b_{t} \\
& +q^{-d_{1}-d_{2}}\left[\frac{\left(1+G_{2} G_{C 2}\right) R_{1}+\overline{G_{1}} R_{2}+T-F_{1} \overline{G_{1}} \overline{G_{2}} G_{C 1} G_{C 2}-S \overline{G_{2}} G_{C 2}\left(1+G_{1} G_{C 1}\right)}{1+G_{2} G_{C 2}+G_{1} G_{2} G_{C 1} G_{C 2}}\right] a_{t} \\
& =\underbrace{F_{b} b_{t}+\left(F_{1}+S q^{-d_{1}}\right) a_{t}}_{\text {controller-independent }}+\underbrace{q^{-d_{1}-d_{2}} L_{b} b_{t}+q^{-d_{1}-d_{2}} L_{1} a_{t}}_{\text {controller-dependent }},
\end{aligned}
$$

where

$$
L_{1}=\frac{\left(1+G_{2} G_{C 2}\right) R_{1}+\overline{G_{1}} R_{2}+T-F_{1} \overline{G_{1}} \overline{G_{2}} G_{C 1} G_{C 2}-S \overline{G_{2}} G_{C 2}\left(1+G_{1} G_{C 1}\right)}{1+G_{2} G_{C 2}+G_{1} G_{2} G_{C 1} G_{C 2}}
$$

and

$$
L_{b}=\frac{\left(1+G_{2} G_{C 2}\right) R_{b}-F_{b} \overline{G_{1}} \overline{G_{2}} G_{C 1} G_{C 2}}{1+G_{2} G_{C 2}+G_{1} G_{2} G_{C 1} G_{C 2}}
$$

are proper. The controller-independent term is independent to the control action and the controller-dependent term. Hence, the variance of the water level output satisfies the following inequality: 


$$
E\left(H^{T} H\right) \geq \operatorname{trace}\left(\operatorname{Var}\left(F_{b} b_{t}\right)\right)+\operatorname{trace}\left(\operatorname{Var}\left(F_{1} a_{t}+S q^{-d_{1}} a_{t}\right)\right) .
$$

The equality holds when $L_{b}=0$ and $L_{1}=0$.We thus have the MV controllers

$$
G_{C 1}=\frac{-R_{b} G_{d 2}}{R_{b}\left(F_{1}+S q^{-d_{1}}\right)-F_{b}\left(R_{1}+T+\overline{G_{1}} R_{2}\right)} \quad G_{C 2}=\frac{\left(F_{1}+S q^{-d_{1}}\right) R_{b}-F_{b}\left(R_{1}+T+\overline{G_{1}} R_{2}\right)}{\overline{G_{2}}\left[-G_{b} S+\left(R_{1} F_{b}-R_{b} F_{1}\right) q^{-d_{2}}\right]}
$$

Hence, the MV performance benchmark can be obtained

$$
\left.H_{t}\right|_{M V}=F_{b} b_{t}+\left(F_{1}+S q^{-d_{1}}\right) a_{t}=F_{b} b_{t}+F_{a} a_{t},
$$

which can be estimated from the routine operating data with some a priori knowledge.

\subsection{Estimation of MV Performance Benchmark of the Drum Water Level Control System}

For the main control loop, a multivariate time series model of $H_{t}$ can be obtained with $a$ priori knowledge of the process time delays. It can be described as the closed-loop transfer functions that relate $\left[a_{t} \xi_{t}\right]$ to $H_{t}$,

$$
H_{t}=\hat{G}_{a} a_{t}+\hat{G}_{\xi} \xi_{t},
$$

where $\hat{G}_{a}$ and $\hat{G}_{\xi}$ are proper and rational transfer function matrices. As above mentioned, we can obtain $\xi_{t}=\hat{G}_{m} b_{t}$ by the time series analysis. Then the equation (8) can be rewritten as

$$
H_{t}=\hat{G}_{a} a_{t}+\hat{G}_{\xi} \hat{G}_{m} b_{t}
$$

Thus, the equation (9) can be represented by the impulse response coefficient,

$$
\begin{aligned}
H_{t}= & \underbrace{\hat{F}_{a, 0} a_{t}+\cdots+\hat{F}_{a, d_{1}+d_{2}-1} a_{t-d_{1}-d_{2}+1}}_{\hat{F}_{a} a_{t}}+\hat{F}_{a, d_{1}+d_{2}} a_{t-d_{1}-d_{2}}+\cdots \\
& +\underbrace{\hat{F}_{b, 0} b_{t}+\cdots+\hat{F}_{b, d_{1}+d_{2}-1} b_{t-d_{1}-d_{2}+1}}_{\hat{F}_{b} b_{t}}+\hat{F}_{b, d_{1}+d_{2}} b_{t-d_{1}-d_{2}}+\cdots
\end{aligned}
$$

The first $d_{1}+d_{2}$ moving average coefficients are all controller-independent terms,

$$
\left.H_{t}\right|_{M V}=\hat{F}_{a} a_{t}+\hat{F}_{b} b_{t}
$$

Furthermore, we have

$$
\begin{aligned}
& \operatorname{Var}\left(\hat{F}_{a} a_{t}\right)=\hat{F}_{a, 0} \Sigma_{a}\left(\hat{F}_{a, 0}\right)^{T}+\cdots+\hat{F}_{a, d_{1}+d_{2}-1} \Sigma_{a}\left(\hat{F}_{a, d_{1}+d_{2}-1}\right)^{T} \\
& \operatorname{Var}\left(\hat{F}_{b} b_{t}\right)=\hat{F}_{b, 0} \Sigma_{b}\left(\hat{F}_{b, 0}\right)^{T}+\cdots+\hat{F}_{b, d_{1}+d_{2}-1} \Sigma_{b}\left(\hat{F}_{b, d_{1}+d_{2}-1}\right)^{T},
\end{aligned}
$$

where $\Sigma_{a}=E\left(a_{t} a_{t}^{T}\right)$ and $\Sigma_{b}=E\left(b_{t} b_{t}^{T}\right)$. Thus, the MV performance benchmark is estimated by

$$
J_{M V}=E\left(\left.\left(\left.H_{t}\right|_{M V}\right)^{T} H_{t}\right|_{M V}\right)=E\left(\left(\hat{F}_{a} a_{t}\right)^{T}\left(\hat{F}_{a} a_{t}\right)\right)+E\left(\left(\hat{F}_{b} b_{t}\right)^{T}\left(\hat{F}_{b} b_{t}\right)\right) .
$$




\subsection{Performance Index of the Drum Water Level Control System}

As suggested in [5], the deviation can be quantified by the performance index based on MV control. The performance index of the drum water level control system can be defined as follows:

$$
\eta=\frac{J_{M V}}{J}=\frac{J_{M V}}{E\left(H_{t}^{T} H_{t}\right)}
$$

This definition of the controller performance index satisfies $0 \leq \eta \leq 1$, and provides a bounded and normalized performance indicator. The distance between minimum variance control system and actual control system can be directly seen. The value $\eta=1$ indicates an ideal case of minimum variance control, whereas $\eta=0$ shows the case of the worst control.

\section{Numerical Example}

As shown in Figure 1 and 2, consider a boiler with $120 t / H$ steam supply. The sampling interval of system is $1 s$. The parameters of the water level control system are

$$
G_{P}=\frac{0.00061 q^{-2}+0.0006 q^{-3}}{1-1.967 q^{-1}+0.9672 q^{-2}}, \quad G_{D}=\frac{0.1274 q^{-1}-0.129 q^{-2}}{1-1.368 q^{-1}+0.3679 q^{-2}},
$$

$\gamma_{H}=1, \gamma_{W}=\gamma_{D}=0.5, K_{Z}=K_{u}=0.8, a_{W}=0.3$ and $a_{D}=0.6$. From Figure 3, we can obtain $d_{1}=2, d_{2}=0, G_{d 2}=1, G_{2}=0.64$,

$$
G_{d 1}=\frac{0.1274 q^{-1}-0.129 q^{-2}}{1-1.368 q^{-1}+0.3679 q^{-2}}, \quad G_{1}=\frac{0.00061 q^{-2}+0.0006 q^{-3}}{1-1.967 q^{-1}+0.9672 q^{-2}} .
$$

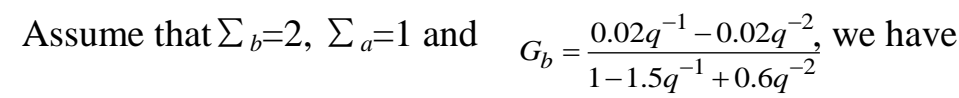

$$
F_{b}=0.02 q^{-1}, F_{1}=0.1274 q^{-1}, F_{2}=1, \quad R_{2}=0, \quad S=0,
$$

$R_{b}=\frac{0.05-0.012 q^{-1}}{1-1.5 q^{-1}+0.6 q^{-2}}, R_{1}=\frac{0.0453-0.0469 q^{-1}}{1-1.368 q^{-1}+0.3679 q^{-2}}, T=\frac{0.00061+0.0006 q^{-1}}{1-1.967 q^{-1}+0.9672 q^{-2}}$.

Thus, the performance benchmark can be obtained as $J_{M V}=0.017$.

Similar to the test method in [6], assume that ${ }_{G_{C 1}}=2-1.99 q^{-1}, G_{C 2}=k$, where $k$ is a variable. When $k=1$, the closed loop response output of the system is shown in Figure 4 (a). According to the different $k$ value, we have different performance index as shown in Figure 4(b). For this form of control, the maximum performance index is about 0.23 when the variable $k$ takes the value of about 0.5 . There may be a possible improvement by enhancing the control performance from the current one to that of the MV performance benchmark.

\section{Conclusions}

A feedforward-cascade drum water level control system is considered in the control loop performance assessment and the control loop performance assessment method is developed. The MV performance benchmark can be estimated by multivariate time series analysis with a priori knowledge of process time delays for the plant. They do not require perturbing the process with extraneous test signals. The numerical example illustrates the effectiveness of the proposed method.

This paper presents a preliminary attempt of performance assessment problem for the boiler drum water level control system. Some future works include applying the proposed 
approach to a more realistic case in practice about a controlled drum water level control system, process identification and so on.

(a)

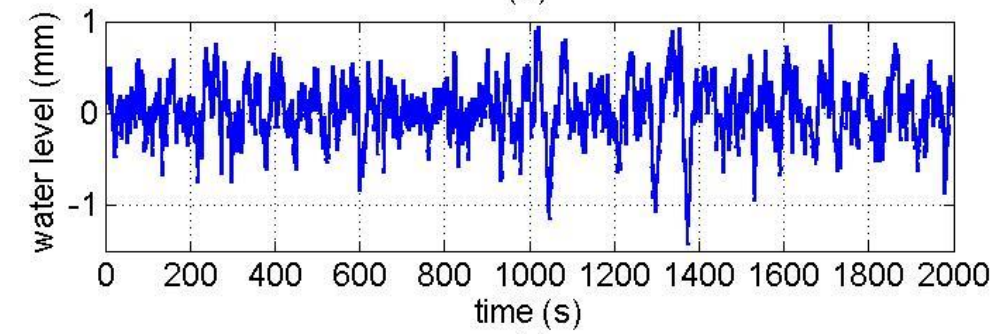

(b)

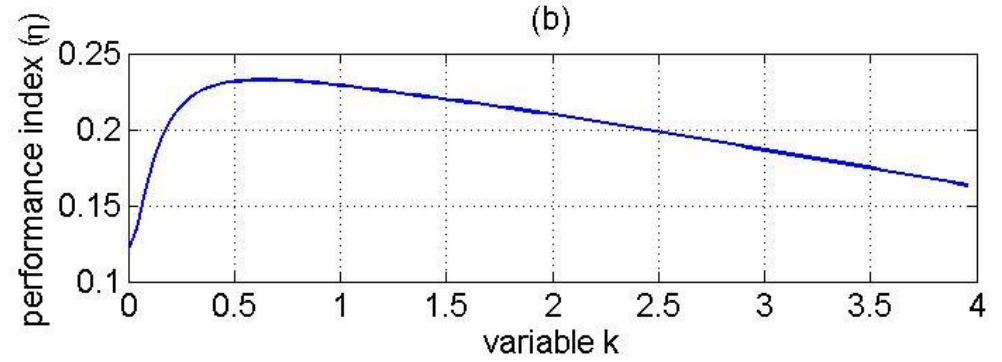

\section{Figure 4. Closed Loop Response Output of the System and Performance Index}

\section{References}

[1] X.-F. Li, "Fuzzy self-adapting PID control of drum water level in a power plant", Proceedings of 1st IFAC Workshop on Convergence of Information Technologies and Control Methods with Power Plants and Power Systems, Cluj-Napoca, Romania, (2007).

[2] J. Wu, K. Jiang, H. R., Karimi and X. Su, "Model predictive control for drum water level of boiler systems", Proceedings of the 26th Chinese Control and Decision Conference, Changsha, China, (2014).

[3] T. J. Harris, C. T. Seppala and L. D. Desborough, "A review of performance monitoring and assessment techniques for univariate and multivariate control systems", J. Process Control, vol. 9, (1999), pp. 1-17.

[4] M. Jelali, "An overview of control performance assessment technology and industrial applications", Control Eng. Pract., vol. 14, (2006), pp. 441-466.

[5] T. J. Harris, “Assessment of closed loop performance”, Can. J. Chem. Eng., vol. 67, (1989), pp. 856861.

[6] B. Huang and S. L. Shah, "Performance Assessment of Control Loops: Theory and Applications", Springer, Berlin, (1999). 
International Journal of Control and Automation Vol. 9, No. 12 (2016) 